\title{
Interactive comment on "On periodic solutions associated with the nonlinear feedback loop in the non-dissipative Lorenz model” by B.-W. Shen
}

\section{Anonymous Referee \#2}

\section{Received and published: 5 December 2016}

This manuscript discusses a model called by the author the non-dissipative Lorenz model. The model is related to the Lorenz 1963 model, but with several terms missing. It is apparently derived from the Boussinesq equations by a method similar to that of Lorenz. The model has a conserved quantity, and for a particular value of the conserved quantity, the model is equivalent to the Duffing equation with no damping and no forcing, i.e., frictionless motion in a double-well potential given by a symmetric 4th-degree polynomial. The analysis of periodic and homoclinic solutions of this model is not novel, and it is not clear to me that it offers significant insight into the physics of the Boussinesq equations.

To be more specific, equation (15) in the manuscript is the Duffing equation discussed above; I find it peculiar that the manuscript does not mention Duffing until Appendix B. The Duffing equation has been studied extensively, and in the particular case of no 
damping and no forcing, its solutions are particularly well understood. The closed-form solution in the special case presented in Appendix B is also derived, for example, in NPGD the discussion below equation (23) of the article at:

http://www.sciencedirect.com/science/article/pii/S0094114X14002079

Closed-form solutions for more general equations are derived in the articles at:

Interactive

comment

http://www.sciencedirect.com/science/article/pii/S0307904X12002302

http://isi-

dl.com/downloadfile/106810

The present manuscript focuses primarily on the analysis of the Duffing model, which I already well understand, and I don't feel that I learned anything from it about the Lorenz or Boussinesq equations.

Interactive comment on Nonlin. Processes Geophys. Discuss., doi:10.5194/npg-2016-40, 2016. 